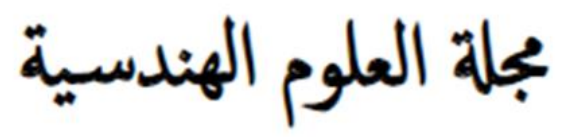

\title{
Prediction of Concrete Compressive Strength \& Slump using Artificial Neural Networks (ANN)
}

\author{
Yasir M. H. Badawi ${ }^{1, *}$ and Yousif Hummaida Ahmed ${ }^{2}$ \\ 1 Civil Engineering Department, Faculty of Engineering \& Technical Studies, Elimam Elmahdi University, Kosti, Sudan \\ 2 Civil Engineering Department, Faculty of Engineering, University of Khartoum, Khartoum, Sudan \\ * Corresponding author: Yasir M. H. Badawi (e-mail: eng.yasirbadawi@gmail.com).
}

Article history: Received 30 March 2020, Received in revised form 4 November 2020, Accepted 11 November 2020

\begin{abstract}
Concrete is the most used building material in the world, due to its high compressive strength and durability. Those properties are measured and assessed in fresh and hardened states of concrete, with standard methods which are time and cost consuming. In the present study, the compressive strength and slump of concrete has been predicted using Artificial Neural Network (ANN), which is constructed using different input parameters involving concrete mix design (i.e. coarse \& fine aggregates properties, cement content, water/cement (W/C) ratio, admixtures type and dosage ...etc.).The predicted strength was compared with the experimentally obtained actual compressive strength and slump data collected in many years for different materials and mix designs in the Sudan. An ANN model has been developed by using MATLAB neural network toolbox. A good co-relationships with regression values of 0.915 and 0.931 for strength and slump respectively have been obtained between the predicted and experimental values. It is concluded that the ANN method can gain acceptable predictions for compressive strength and slump.
\end{abstract}

Keywords: Artificial Neural Network (ANN), Compressive strength, Mix design, Modelling, Regression, Slump.

\section{INTRODUCTION}

Acquiring concrete mix design is a sensitive and difficult process. It is affected by several parameters, yet one mixture design is effective only for one compressive strength. In every production that involves variations in material characteristics that are slightly different, new mixture design is necessary. This led to the conventional mixture design results being inaccurate. In the end, it shows that manual mixed design is merely an estimation[1].

Methods of mix design can be classified into empirical methods and methods based on artificial intelligence (AI). The former includes methods that are developed empirically with regard to experience such as methods of American Concrete Institute (ACI), and the British Department of Environment (DoE). They involve determining quantities of ingredients by different ways.

Methods of artificial intelligence are computer-based techniques, which are developed based on the concept of machine learning, ANN, Fuzzy analysis and Genetic algorithm are the most common methods[2].

The ANNs are computational models that adopt a training mechanism to extract the relationships that link a set of causal input parameters to the resulting conclusions. Once ANNs are trained, they can predict the results for an unknown case (not used in training) if provided with the input parameters alone.

In this paper a model of ANN has been constructed to predict concrete compressive strength and slump using data of different mix designs. The model is validated, tested, and regression analysis has been carried out to 
evaluate the accuracy of the model. At first the data has been collected, then preprocessed (i.e. reviewing, validating data, standardization and normalization ...etc.). And then the model is constructed.

\section{STATE OF THE ART}

ANN offers an alternative to mathematical modelling. The main concept of the neural network is to feed it with input data and target output data, and it will learn the relationship between input and output. Subsequently, the trained network can be used to predict the outcome of other sets of input, where the answer is unknown. For further explanation of ANN technique, refer to introductory textbooks on the subject in reference [3]or [4].

Using ANN has many advantages. includes solving complex problems for which doesn't exist any sequential algorithms. Instead there are only examples of solutions. The ability of the ANNsto adapt to a changing environment; deterioration of some neurons does not involve a steep deterioration in performance, but degrades network performance. The ANNs exhibit opportunity to work with imprecise data; ability to modify the internal structure in order to perform the desired action. They generate their own rules of learned examples and are used to model the nonlinear systems. Creating a well-trained ANN lead to the removal experimental phase; inexpensive and fast to a slow and expensive program structural analysis. They may be used for real-time applications; ability to approximate a nonlinear continuous function with the desired degree of accuracy; easily modeled neural networks multivariable systems (due to the large number of inputs and outputs).

Among the disadvantages noted for the ANNs are: the learning process is complicated because of the difficulty in choosing the training set. Training is lengthy depending on the training's method and the training set size and requires very efficient computing means. For training it takes a very large data volume; establish training base is a difficult operation, it must cover all the search satisfactory solution[2].

The ANN has the same weakness as the mathematical model. Because it is also based on empirical data. But the advantage over the mathematical model is that the programmer making the model doesn't have to declare every action of the program. When using ANN applications for problem solving, it is needed to understand the problem to such a level that relevant parameters can be chosen as input. But when the network is trained, then learning about the problem is achieved by studying the way the network generalizes. In other words, the neural network summarizes the experience hidden in the input-output relationship.

The ANN has been called the second-best way to do just about anything [1]. The best way is of course to attain a full understanding of the problem and then find the right formula or optimum algorithm for the problem. However, this may not always be possible, and it leaves plenty of problems to be solved by the second-best approach[5].

\section{COMPUTER IMPLEMENTATION OF THE NEURAL NETWORK MODEL}

\section{A. Data description}

Data used in model has been tabulated, organized, filtered and published by researchers [6]. The data includes mix designs according to the British DoE, the parameters used are illustrated in Table I.

\begin{tabular}{|c|c|c|c|}
\hline & Parameter & Type & Designation \\
\hline 1 & Type of coarse Aggregates & Input & $\mathrm{X} 1$ \\
\hline 2 & Type of Fine Aggregates & Input & $\mathrm{X} 2$ \\
\hline 3 & $\begin{array}{l}\text { Max. Size of Coarse Aggregate } \\
(\mathrm{mm})\end{array}$ & Input & $\mathrm{X} 3$ \\
\hline 4 & Sand Passing $0.6 \mathrm{~mm}$ Sieve (\%) & Input & $X 4$ \\
\hline 5 & $\begin{array}{l}\text { Ordinary Portland Cement } \\
(\mathrm{OPC})\left(\mathrm{kg} / \mathrm{m}^{3}\right)\end{array}$ & Input & X5 \\
\hline 6 & W/C Ratio & Input & X6 \\
\hline 7 & Water Content $\left(\mathrm{kg} / \mathrm{m}^{3}\right)$ & Input & $\mathrm{X7}$ \\
\hline 8 & Admixture class & Input & $\mathrm{X} 8$ \\
\hline 9 & Admixture dosage (Litre) & Input & X9 \\
\hline $\begin{array}{l}1 \\
0\end{array}$ & Fine Aggregate $\left(\mathrm{kg} / \mathrm{m}^{3}\right)$ & Input & $\mathrm{X} 10$ \\
\hline $\begin{array}{l}1 \\
1\end{array}$ & Coarse Aggregate $\left(\mathrm{kg} / \mathrm{m}^{3}\right)$ & Input & $\mathrm{X} 11$ \\
\hline $\begin{array}{l}1 \\
2\end{array}$ & $\begin{array}{l}\text { Consistence (i.e. Workability) } \\
\text { measured by Slump (mm) }\end{array}$ & Output & Y1 \\
\hline $\begin{array}{l}1 \\
3\end{array}$ & Strength $\left(\mathrm{N} / \mathrm{mm}^{2}\right)$ (7 days avg.) & Output & $\mathrm{Y} 2$ \\
\hline
\end{tabular}

\section{B. Construction of Neural Network model:}

For a feed-forward back-propagation network structure and training process, the important 
internal parameters include data preprocessing and presentation and initial synaptic weights. Furthermore they include learning rate, number of hidden layers and number of neurons in each hidden layer, activation functions for hidden layers and output layers and the number of training epochs [7].

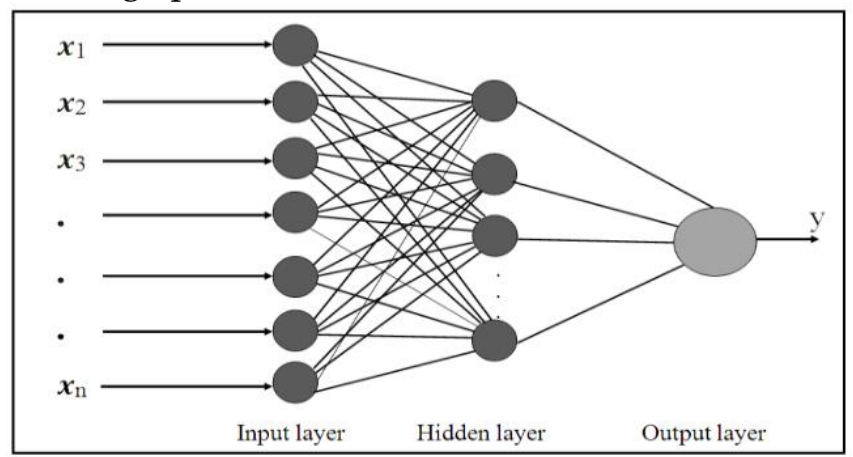

Fig. 1: ANN structure

In this work, a three-layer feed-forward back-propagation neural network is developed through experimental investigation of various internal parameters to predict the slump and compressive strength of concrete.

In Fig. 1: $\mathrm{X} 1, \mathrm{X} 2, \mathrm{X} 3, \ldots . ., \mathrm{Xn}$ are the input variables, where for the problem in hand, the values is defined as in Table I.

The model is constructed to predict the properties of fresh concrete (i.e. Slump), and also to predict the properties of hardened concrete (i.e. strength in 7 days). With the same input data and modelling parameters.

\section{Modelling parameters:}

Selection of activation function, Number of neurons in hidden layer, training algorithm and type of networks can influence the effective functioning of the ANNS approach [8].

According to literature [9], the most used types of activation functions are: Sigmoid functions (tan sigmoid and log sigmoid) and Purelin function (linear function).

Number of hidden neurons: The hidden layer and nodes play very important roles for many successful applications of neural networks. It is the hidden nodes in the hidden layer that allow neural networks to detect the feature, to capture the pattern in the data, and to perform complicated nonlinear mapping between input and output variables. There is no theoretical basis for selecting this parameter although, so optimization is carried out by selecting different numbers of hidden neurons (from 10 to 100 hidden neurons) and choosing the optimum number that has the smallest mean squared error (MSE), and the largest regression coefficient.

Division of data: The issue of data division into training, validation and test sets is one of most affecting factors. There is no general solution to this problem, several factors such as the problem characteristics, the data type and the size of the available data should be considered in making the decision. It is critical to have both the training, validation and test sets representative of the population or underlying mechanism.

Inappropriate separation of the training and test sets will affect the selection of optimal ANN structure and the evaluation of ANN forecasting performance. The literature offers little guidance in selecting the training and the test sample. Most authors select them based on the rule of $70 \%$ for training, 20\% for validation and 10\% for testing.

The architecture of the two developed ANNs in this paper are abbreviated as (STR-11-n-1) and (CON11-n-1) with STR denoting strength and CON denoting consistence.

The MSE was used as the ANN stop training criterion. In this regard, lower values are corresponding to more idealized network performance. Regression values (R-values) are utilized to measure the correlation between outputs and targets in the networks wherein an $\mathrm{R}$-value of unity indicate strong relationships. The MSE and R-values were applied as the criteria for evaluation of the generated networks performance.

\section{RESULTS \& DISCUSSION}

\section{A. Implementation results:}

The eleven input parameters $(\mathrm{X} 1 \sim \mathrm{X} 11)$ as shown in Table I, are utilized as the input layer with one hidden layer in the architecture of the ANN model (Fig. 1). All nodes in the ANN model utilize the log-sigmoid function as their activation. Two ANNs were constructed, one for predicting the strength at 7 days, and the other for predicting the consistence as slump.

For training the ANNs, 1017 samples were randomly divided into training (712 samples), validation (203 samples) and test sets (102). The training set wasused to teach the network. Training had continued as long as the network 
improved on the validation set. The test set provided a completely independent measure of network accuracy[10].

The network type utilized in this study was the Back-propagation ANN. A typical neuron in the network contains biases, a sigmoid activation function and a linear output layer. It is capable of

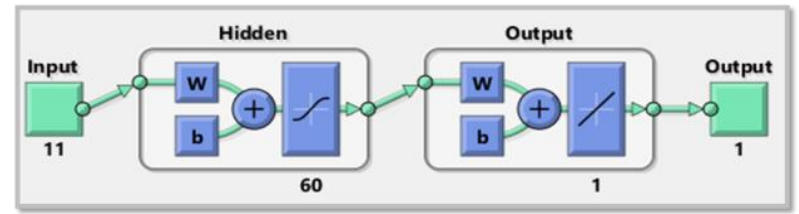

Fig. 2: General layout of the ANN

approximating any function having a finite number of discontinuities as illustrated in Fig. 2.

The results of training were as shown in Figs 3, 4, 5 \& 6:
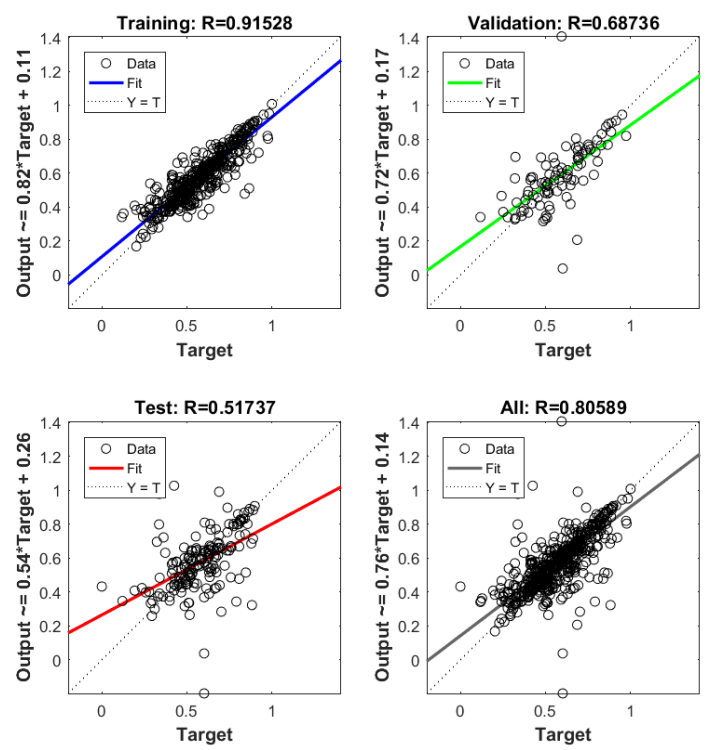

Fig. 3: Regression of training, validation and test simulated by STR11-60-1.
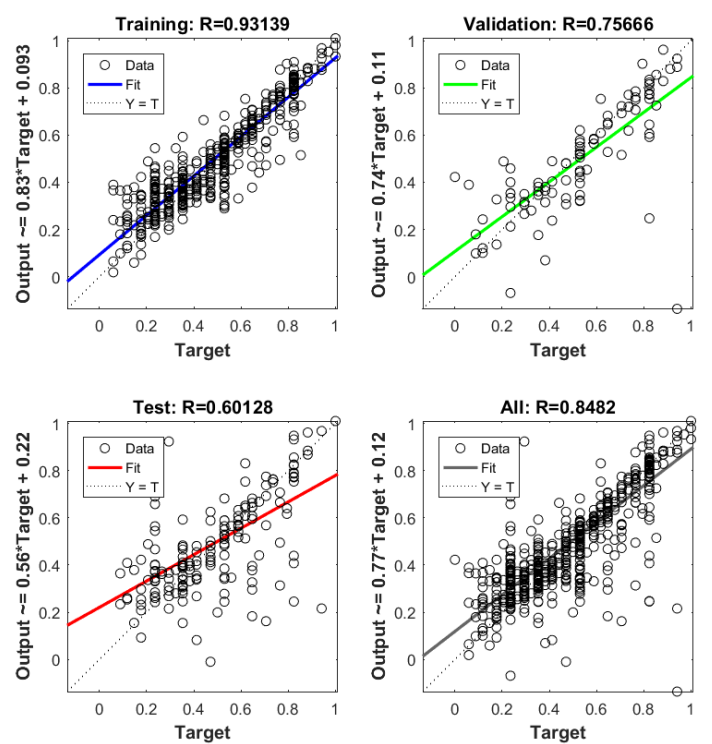

Fig. 4: Regression of training, validation and test simulated by CON11-60-1.

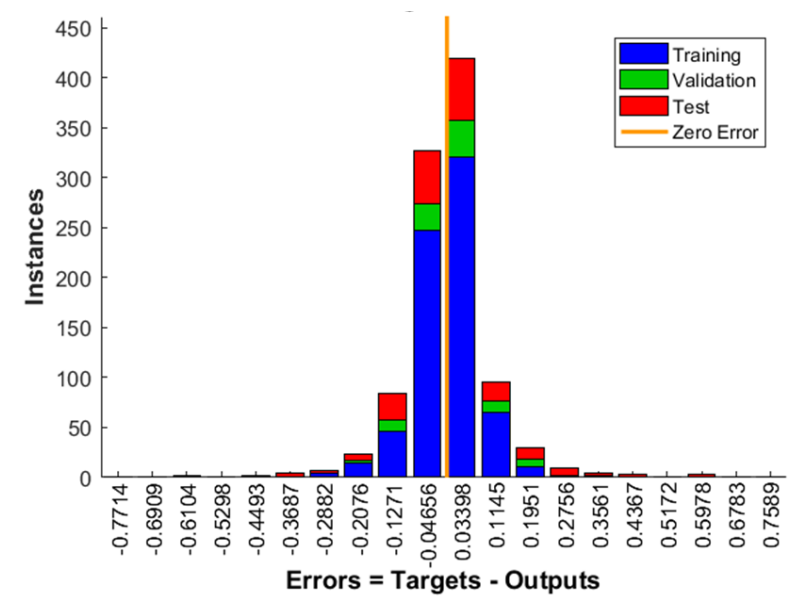

Fig. 5: Error histogram for STR11-60-1

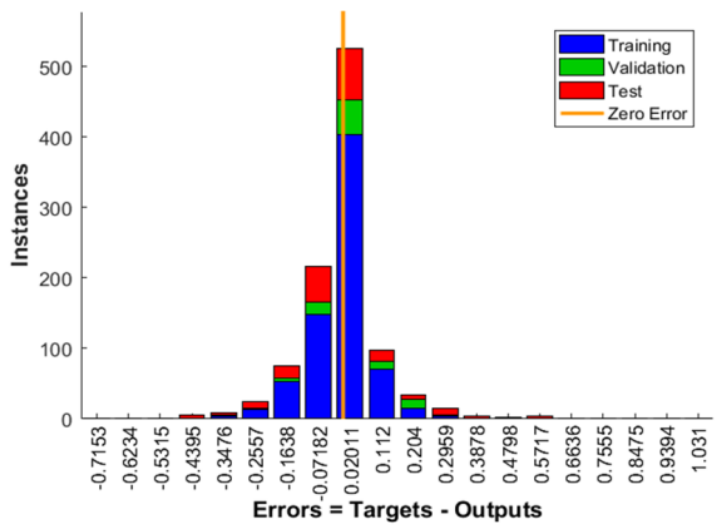

Fig. 6: Error histogram for CON11-60-1

\section{B. Optimization}

The optimization was carried out by implementing 10 networks with different number of hidden neurons in each time, the number of hidden neurons is plotted against regression coefficient in Fig. 7 \& Fig. 8that show R-values of the networks having various numbers of hidden nodes.

For STR11-n-1 the highest R-value for training data was obtained with 100 hidden neurons and 60 hidden neurons, (they are 0.915 and 0.911 respectively). However, CON11-n-1 the highest R-value for training data was obtained with 60 hidden neurons and 50 hidden neurons (which are 0.931 and 0.921 respectively).

\section{Results of optimized ANNs}

Generally, the error reduces after more epochs of training, but might start to increase on the validation data set as the network starts overfitting the training data. In the default setup, the training stops after six consecutive increases in validation error, and the best performance is taken from the epoch with the lowest validation error. 


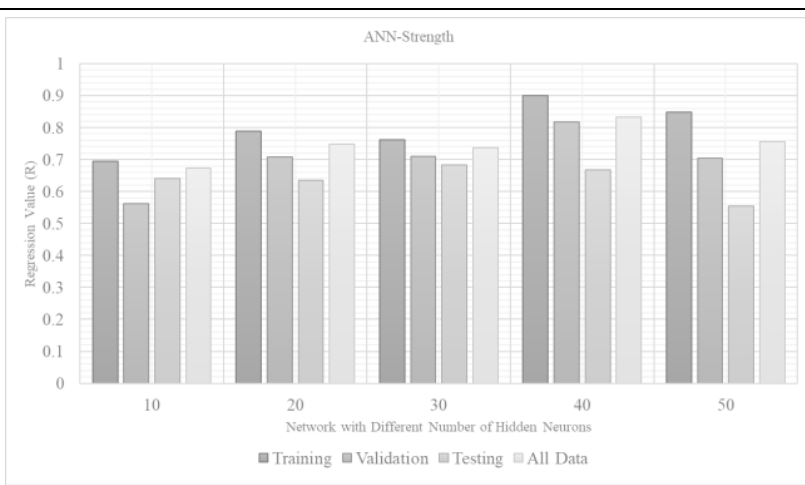

(a)

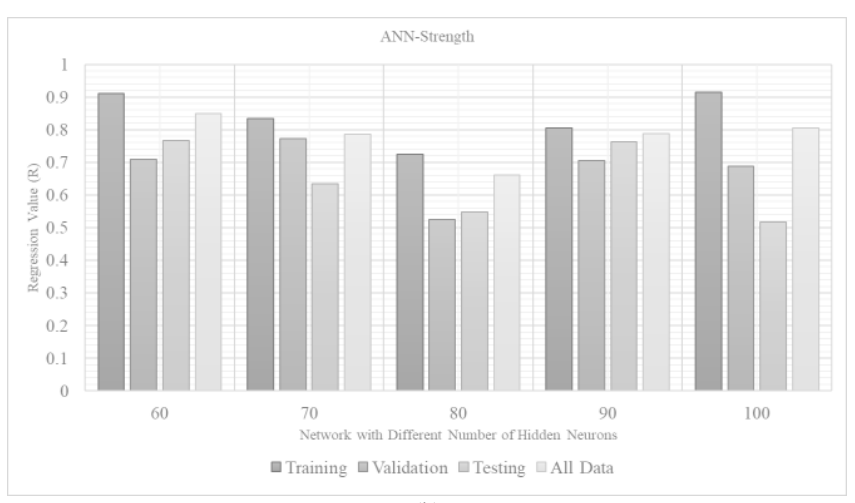

(b)

Fig. 7 Effect of number of hidden neurons on regression coefficient for STR 11-n-1.

Another measure of how well the neural network has fit the data is the regression plot (Fig. 3 \& Fig. 4). Here the regression is plotted across all samples, the regression plot shows the actual network outputs plotted in terms of the associated target values. If the network has learned to fit the data well, the linear fit to this output-target relationship should closely intersect the bottom-left and top-right corners of the plot[10].

Another third measure of how well the neural network has fit data is the error histogram (Fig. 5 \& Fig. 6). This shows how the error sizes are distributed. Typically, most errors are near zero, with very few errors far from that.

As it can be seen, it may be concluded that the ANN model learnt and predicted the experimental data with acceptable degree of precision for strength in 7 days and slump, but there is low precision and high errors in some cases. This can be interpreted by the effect of admixture, probably because of the different types of admixtures with different specifications and manufacturers, which led to variability in some results.

\section{SUMMARY}

There are not enough investigations dealing with development of fundamental knowledge which leads to understand the nature of the internal representations generated by an ANN in response to a given problem. More than often, an ANN is presented to its users as a black box with complicated internals which work to convert inputs into desirable outputs.

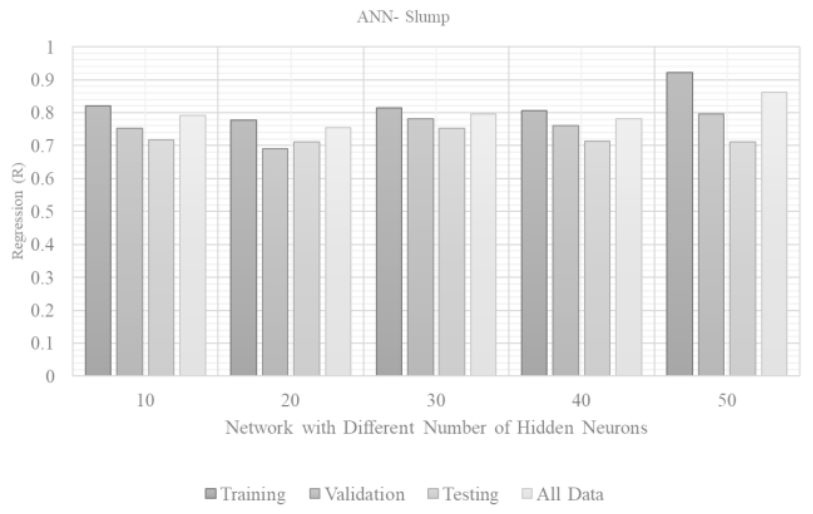

(a)

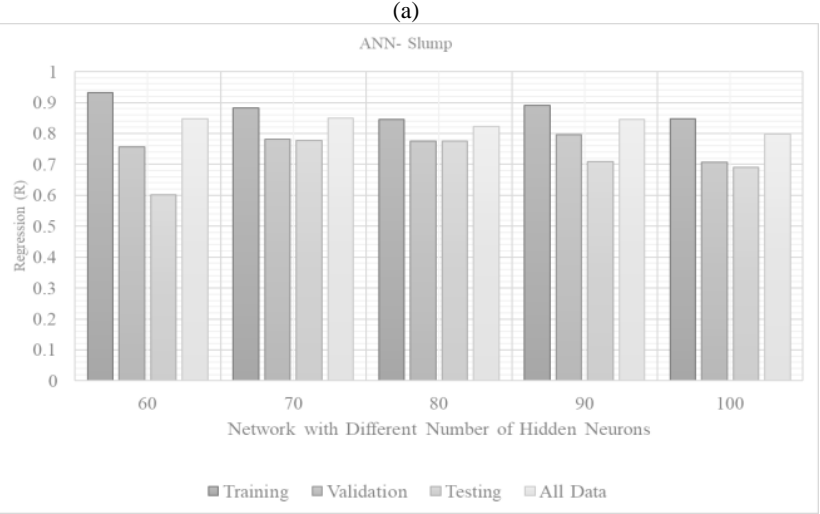

(b)

Fig. 8 Effect of number of hidden neurons on regression coefficient for CON11-n-1.

In this study, an ANN is developed to evaluate the slump and strength at 7 days for data collected in many years from different locations in Sudan. The regression values of the chosen strength network for training is 0.915 . And for the chosen slump network is 0.931 for training. The best validation performance was observed in epoch 8 for STR11-60-1 and epoch 10 for CON11-60-1.

It is concluded that the ANN method is capable of gaining acceptable predictions for compressive strength and slump.

\section{Recommendations}

- Further research in this field should be carried out to study the effect and importance of each parameter in the results of the ANN model.

- Many important factors like, fineness modulus of aggregates, shape of aggregates, absorption 
and specific gravity which have great effect in strength of concrete, further studies should take into account the variability of these factors.

- The admixtures used in the data sets are variable in types and manufacturers, the dose of each product differs from company to company, but in this study, it is assumed that all the admixtures fall in the same ASTM classification, have the same properties. This assumption affected the slump prediction accuracy of the model, because the majority of the admixtures are related to consistence.

- The package used in this study is MATLAB Neural Network toolbox, further research may use other packages to compare the results and increase their reliability.

\section{ACKNOWLEDGMENT}

The authors thankfully acknowledges Eng. Khalid Mustafa, Ahmed Sultan for their valuable support, and also the technical support from the University of Khartoum materials laboratory staff, especially Eng. Yahya A. Idris and Eng. Abdulrahim Mohamed Yousif. The author also thanks Dr. Amjad Osman et al. for their great effort in data collection, organizing and entry.

\section{REFERENCES}

[1] Santosa S, Santosa YP. Evolutionary Artificial Neural Networks for Concrete Mix Design Modelling. Int J Comput Appl. 2017;7(5):62-70.

[2] Pandelea A-EC, Budescu MG, Covatariu GM. Applications of Artificial Neural Networks in Civil Engineering. In: Proceedings of the Second International Conference for PhD Students in Civil Engineering and Architecture. 2014.

[3] Hertz J, Krogh A, Palmer RG. Introduction to the theory of neural computation. Addison-Wesley/Addison Wesley Longman; 1991.

[4] Skapura DM. Building neural networks. Addison-Wesley Professional; 1996.

[5] Jepsen MT. Predicting concrete durability by using artificial neural network. In: Durability of Exposed Concrete containing Secondary Cementitious Materials. 2002. p. 1-12.

[6] Abdelatif AO, Shaddad AMY, Fathallah MB, Ibrahim MS, Twfeeq MH. Concrete mix design and aggregate tests data between 2009 and 2017 in Sudan. Data Br [Internet]. 2018;21:146-9. Available from: https://linkinghub.elsevier.com/retrieve/pii/S2352340918311399

[7] Sharma AK, Sharma RK, Kasana HS. Prediction of first lactation 305-day milk yield in Karan Fries dairy cattle using ANN modeling. Appl Soft Comput. 2007;7(3):1112-20.

[8] Paul SC, Panda B, Garg A. A novel approach in modelling of concrete made with recycled aggregates. Measurement [Internet]. 2018;115(October 2017):64-72. Available from: http://dx.doi.org/10.1016/j.measurement.2017.10.031

[9] Naderpour H, Hossein A, Fakharian P. Compressive strength prediction of environmentally friendly concrete using arti fi cial neural networks. J Build Eng. 2018;16(October 2017):213-9.
[10] Demuth H, Beale M, Hagan M. Neural Network Toolbox TM 6 User' s Guide [Internet]. 15 edition. Natick: The MathWorks, Inc.; 2009. Available from: www.mathworks.com/contact_TS.html

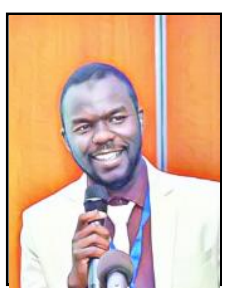

Yasir M. H. Badawi.

He earned his Bachelor of science (honors) in Civil engineering at University of Khartoum in Khartoum, Sudan, in 2014, and his Master of science in Structural engineering from the University of Khartoum, in 2018.

After gaining bachelor degree he worked in University of Elimam Elmahdi, Kosti as teaching assistant. In November 2015 he attended China-Sudan Symposium on advance Material \& Technique in Civil engineering 2015. In December 2016 he attended the first conference of civil engineering held at University of Abdulatif alhamad (Merwe), Sudan as representative of University of Elimam Elmahdi. In the 2nd conference of civil engineering (CCE18) which held at University of Khartoum in December 2018, he presented a research paper titled: “Use of Maturity Sensors to predict Concrete Compressive Strength in Different Curing and Compacting regimes".

After he awarded the master degree he worked as part-time lecturer at University of Science and Technology, Omdurman, Sudan. And also, he is a full-time lecturer at University of Elimam Elmahdi.

Yasir M. H. Badawi is also has graduate membership in Sudanese engineering council. He works also as structural designer.

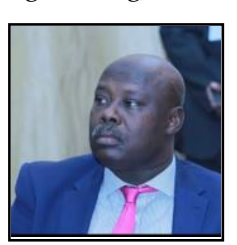

Dr Yousif Hummaida Ahmed (PhD , DIC, $\mathrm{BSc}), \mathrm{PhD}$ in Engineering from Imperial College/University of London (1996).

Awarded Dr Shawki Saad Scholarship. Specialization concrete durability \&hazardous waste management technologies former Head of Civil Engineering Department, Faculty of Engineering ,University of Khartoum ,Sudan. Currently Chairman of the governmental Council for Registering and Classifying Engineering Works Contractors. 